\title{
Corela
}

Cognition, représentation, langage

HS-12 | 2012

Langue, espace, cognition

\section{Evénements et localisation spatiale : quand le lieu est un événement}

\section{Richard Huyghe}

\section{(2) OpenEdition}

\section{Journals}

\section{Édition électronique}

URL : http://journals.openedition.org/corela/2786

DOI : $10.4000 /$ corela.2786

ISSN : 1638-573X

\section{Éditeur}

Cercle linguistique du Centre et de I'Ouest - CerLICO

\section{Référence électronique}

Richard Huyghe, « Evénements et localisation spatiale : quand le lieu est un événement ", Corela [En ligne], HS-12 | 2012, mis en ligne le 12 avril 2013, consulté le 30 avril 2019. URL : http:// journals.openedition.org/corela/2786 ; DOI : 10.4000/corela.2786

Ce document a été généré automatiquement le 30 avril 2019

\section{(c) (i) (2)(2)}

Corela - cognition, représentation, langage est mis à disposition selon les termes de la licence Creative Commons Attribution - Pas d'Utilisation Commerciale - Partage dans les Mêmes Conditions 4.0 International. 


\title{
Evénements et localisation spatiale : quand le lieu est un événement
}

\author{
Richard Huyghe
}

1 Contrairement à leur relation au temps, la relation des événements à l'espace peut sembler confuse. Elle a fait l'objet d'analyses contradictoires dans les travaux de philosophie du langage consacrés aux événements. Des auteurs comme Vendler (1967) défendent l'idée que les événements n'ont qu'une relation indirecte à l'espace, arguant du fait que les événements n'ont pas d'étendue spatiale. D'autres, comme Davidson (1967), Quinton (1979) ou Hacker (1982), admettent que les événements sont des entités spatiales, mais divergent sur leurs propriétés spatiales, notamment en ce qui concerne la délimitation des événements dans l'espace. Ces débats confirment l'idée que, du point de vue ontologique, «la structure spatiale [des événements] n'est pas aussi facile à appréhender que celle d'autres entités » (Casati \& Varzi 1999 : 7).

Qu'en est-il du traitement linguistique des événements? Tels qu'ils sont dénotés par les expressions nominales correspondantes, les événements se présentent comme des entités dotées d'un ancrage spatial (cf. Huyghe 2009). Nous considérons ici comme GN événementiel (désormais GNev) tout GN qui peut être sujet de avoir lieu (cf. Balibar-Mrabti 1990, Gaatone 1992, Godard \& Jayez 1996, Gross 1996) ${ }^{1}$, comme dans :

(1) (La réunion / la conférence de presse du ministre des affaires étrangères / un entretien avec le candidat/ le tournage du reportage/ le vernissage/ une manifestation contre le plan d'austérité / l'épreuve écrite / un concert de Kurt Wagner / l'audition du témoin) a eu lieu dans la matinée.

3 Or les GNev peuvent, à l'instar des GN dénotant des êtres et des objets, désigner l'entité localisée (ou cible) relativement à un repère de localisation donné (le site) dans des phrases thétiques de localisation spatiale, introduites par Il y $a$ :

(2) Il y a (un homme / un cerisier / un hamac / une cabane) dans le jardin.

(3) Il y a (une réunion / un concert / un vernissage / une conférence de presse) dans le hall de l'hôtel.

4 Van de Velde, qui défend l'idée que les événements « sont directement dans l'espace et dans le temps» (2006: 186), fait valoir que les noms d'événements peuvent aussi bien figurer dans la question Où est le $N$ ? que dans Quand est le $N$ ?: 
(4) Où est (la réunion / le concert / la conférence de presse) ?

(5) Quand est (la réunion / le concert / la conférence de presse) ?

Autre indice de leur dénotation spatiale, les GNev peuvent, sous certaines conditions, exprimer le lieu, dans des phrases de localisation statique ou dynamique :

(6) Où est Pierre? - Il est (à un colloque sur les adverbes / à une réunion des alcooliques anonymes / au concert de Kurt Wagner).

(7) Pierre va (au mariage de son collègue / au match France-Argentine / à la conférence de presse du ministre des affaires étrangères).

6 Nous souhaitons revenir ici sur les propriétés descriptives spatiales des GNev, notamment sur l'idée qu'ils dénotent des entités spatiales d'un genre particulier (cf. Huyghe 2011). Les GNev se distinguent des GN dénotant des êtres et des objets (désormais GNobj), par le fait qu'ils ne décrivent pas des cibles et sites spatiaux prototypiques, en raison notamment de leurs propriétés de description temporelle. Par ailleurs, le critère spatial fait apparaître des disparités au sein de la classe des GNev, et peut en ce sens étayer la distinction entre différents types de GNev. Ainsi espérons-nous mettre en évidence (i) la spécificité et (ii) l'hétérogénéité des GNev quant à la dénotation d'entités spatiales. En arrière-plan de ce travail, outre une contribution à la description sémantique des noms d'événements, figurent des interrogations sur le caractère concret ou abstrait des GNev, sur les fondements sémantiques de la distinction entre objets et événements - s'agit-il d'une différence d'ordre spatial ? - et sur le rapport entre les propriétés nominales de description spatiale et temporelle.

\section{Différents modes de localisation spatiale}

L'apparent parallélisme des exemples (2)-(3) ne doit pas masquer certaines différences fondamentales entre GNev et GNobj, s'agissant de la dénotation de cibles spatiales. A bien des égards, l'expression de la localisation dans l'espace n'est pas la même pour les GNev et les GNobj. La différence de traitement apparaitt dans le choix des noms de sites associés aux deux types de GN, et dans les verbes employés pour introduire la localisation spatiale.

\subsection{Construction avec les noms généraux d'espace}

GNev et GNobj se combinent avec des noms généraux d'espace (désormais NGE) distincts (cf. Huyghe 2009). Les NGE (e.g. lieu, endroit, place, zone, site, région) sont des noms du lexique spatial dénués de traits positionnels, directionnels et géométriques, et qui décrivent essentiellement des sites de localisation spatiale. Leurs compléments en de peuvent désigner des cibles spatiales, en relation avec le nom tête. Les GNobj se construisent de cette manière avec le nom place, tandis que les GNev sélectionnent le nom lieu :

(8) (la place / *le lieu) de Marion, (la place / *le lieu) de la lampe, (la place / *le lieu) de l'armoire

(9) (le lieu / *la place) de la réunion, (le lieu / *la place) de la cérémonie, (le lieu /

*la place) du concert

La particularité de place est d'indiquer un schéma d'occupation de l'espace, i.e. de saturation potentielle de l'étendue par l'entité localisée. Le site se présente comme une portion d'espace exclusivement mobilisée par la cible. La mise en profil par place de l'étendue spatiale explique notamment ses emplois massifs (cf. Franckel 1993, Vandeloise 
2001) : une phrase comme Il y a de la place ici renvoie à une certaine quantité continue d'espace vide que l'on peut combler.

L'incompatibilité des GNev avec place corrobore l'idée que les événements ne se voient pas directement associer d'extension spatiale. De fait, contrairement aux GNobj, les GNev se construisent difficilement avec les expressions décrivant l'étendue spatiale. Ils rejettent notamment les compléments de mesure spatiale en de :

(10) a. une maison de cent mètres carrés, une lampe de trente centimètres de haut, un champ de trente hectares

(10)b. *une réunion de trente mètres carrés, *un concert de cinquante mètres de large, ${ }^{*}$ une cérémonie de dix hectares

11 Le nom lieu pour sa part indique, dans ses emplois distinctifs, une localisation qui s'accompagne de la réalisation d'un procès, comme en témoignent les expressions de la forme lieu de + nom d'activité :

(11) un lieu de (travail / méditation / formation / discussion / promenade / recherche)

12 Avec lieu, l'espace n'est pas envisagé comme un réceptacle, mais comme un cadre d'action. Le nom n'a pas d'emplois massifs; il véhicule une conception discontinue de l'espace, qui est structuré par ce qui s'y passe plutôt que par ce qui s'y trouve. Les GNobj ne décrivant pas des entités dynamiques, ils sont exclus de la tournure le lieu du / de la + nom de cible (cf. (8)).

13 L'espace de localisation s'appréhende donc différemment selon qu'on envisage le ciblage des événements ou des objets. On notera que, du point de vue de la description linguistique, l'ancrage spatial n'implique pas l'étendue spatiale. Réduire les critères de spatialité à la seule extension spatiale ne permet pas de prendre en compte l'ensemble des entités dotées de propriétés spatiales.

\subsection{Construction avec les prédicats introduisant la localisation}

Les verbes employés pour introduire la localisation spatiale diffèrent également, selon que les cibles sont dénotées par des GNobj ou des GNev. Les GNobj se construisent avec se trouver, les GNev avec avoir lieu (cf. Godard \& Jayez 1996, Kleiber et al. à paraitre) :

(12)a. Pierre (se trouve / *a lieu) à Helsinki.

(12)b. Ce livre (se trouve / *a lieu) sur l'étagère.

(13)a. Ce colloque (a lieu / *se trouve) à Paris.

(13)b. L'avant-première du film (a lieu / *se trouve) dans une salle d'art et d'essai.

Avoir lieu se distingue de se trouver par le fait qu'il exprime la réalisation de quelque chose. Il peut s'employer sans complément :

(14)a. La rencontre entre les deux hommes a (effectivement) eu lieu.

(14)b. Cette conversation a (effectivement) eu lieu.

(15)a. *Pierre se trouve (effectivement).

(15)b. *''armoire se trouve (effectivement).

16 Contrairement à se trouver, avoir lieu n'est pas strictement dédié à la prédication locative. Avoir lieu ne se contente pas d'introduire la localisation spatiale, mais il sert à poser l'existence d'un sujet de nature dynamique. $X$ a eu lieu à tel endroit contient deux informations : (i) la réalisation de $\mathrm{x}$ et (ii) la localisation de $\mathrm{x}$ à tel endroit. Présupposant la dynamicité du sujet, avoir lieu associe la localisation de la cible à son actualisation dans l'espace-temps. 

Il est intéressant de constater qu'il n'existe pas de pur prédicat de localisation spatiale pour les GNev. Cela peut s'expliquer par la nature dynamique des événements, qui implique une structure temporelle, et fait que l'ancrage spatial des événements dépend étroitement de leur ancrage temporel. De fait, avoir lieu, contrairement à se trouver, introduit indifféremment la localisation spatiale et temporelle. Un SP de datation ne peut à lui seul compléter se trouver :

(18)a. La réunion a lieu dans la salle du conseil.

(18)b. La réunion a lieu à $14 \mathrm{~h}$.

(19)a. Le tableau se trouve dans la salle du conseil.

(19)b. * Le tableau se trouve à $14 \mathrm{~h}$. deux constituants de temps et de lieu :

(20)a. Le tableau se trouvait dans la salle du conseil à $14 \mathrm{~h}$.

(20)b. La réunion a eu lieu dans la salle du conseil à $14 \mathrm{~h}$. adjoint qui s'applique à l'ensemble de la proposition Le tableau se trouvait dans la salle du conseil. Cette hiérarchie ne se retrouve pas avec avoir lieu dans (20b), où les constituants spatial et temporel sont tous deux des adjoints.

La (dis)symétrie entre compléments de lieu et de temps, selon qu'ils s'associent à des GNev ou à des GNobj, se retrouve dans les phrases thétiques introduites par Il y a. En effet, Il y a peut se contenter d'un complément de temps s'il est suivi d'un GNev, mais pas d'un GNobj :

(21)a. Il y a une réunion dans la salle du conseil.

(21)b. Il y a une réunion à $14 \mathrm{~h}$.

(22)a. Il y a un tableau dans la salle du conseil.

(22)b. *Il y a un tableau à $14 \mathrm{~h}$.

Les GNev se distinguent donc des GNobj par la description d'entités directement ancrées dans le temps. Cet ancrage temporel direct conditionne l'expression de la localisation spatiale. Ainsi les phrases de localisation spatiale mobilisent-elles beaucoup plus facilement des temps grammaticaux accomplis avec les GNev qu'avec les GNobj :

(23)a. Il y a une cérémonie en hommage aux victimes devant l'Hôtel de ville.

(23)b. Il y a eu une cérémonie en hommage aux victimes devant l'Hôtel de ville. 
(24)a. Il y a un inconnu dans le hall.

(24)b. (?)Il y a eu un inconnu dans le hall.

Certains GNev, parce qu’ils dénotent des événements ponctuels ou imprévus (cf. §3.2.), privilégient même fortement les temps accomplis, que ce soit avec Il y a ou avoir lieu. Les événements en question sont généralement constatés une fois qu'ils se sont réalisés :

(25)a. Il y a une avalanche au Grand Ballon d'Alsace (-)

(25)b. Il y a eu une avalanche au Grand Ballon d'Alsace (+)

(26)a. Il y a un choc terrible entre les deux véhicules (-)

(26)b. Il y a eu un choc terrible entre les deux véhicules (+)

(27)a. Un déraillement a lieu sur la ligne Paris-Brest (-)

(27)b. Un déraillement a eu lieu sur la ligne Paris-Brest (+)

(28)a. Un miracle a lieu à Faverney (-)

(28b. Un miracle a eu lieu à Faverney $(+)$

Cette prédilection pour les temps accomplis explique l'impossibilité d'employer certains noms d'événements dans la question Où est le $N$ ?:

(29) Où est (la réunion / la fête / le match / le colloque / le feu d'artifice / le mariage / l'assemblée générale / le festival) ?

(30) *Où est (le déraillement / l'avalanche / la collision / l'explosion / le meurtre /

l'altercation / le massacre / le décès) ?

Aux GNev et aux GNobj correspondent donc deux expressions de l'ancrage spatial différentes. La spécificité des cibles événementielles tient notamment à leur relation directe au temps (cf. Huyghe 2011).

\section{Les GNev en complément de lieu}

La dépendance entre les propriétés descriptives spatiales et temporelles des GNev apparait dans leur emploi comme complément de lieu, tel qu'il est illustré dans les exemples (6)-(7). En effet, les compléments de lieu construits à partir des GNev mettent en jeu une composante temporelle inhérente aux GNev, y compris lorsque celle-ci n'est pas convoquée syntaxiquement - i.e. lorsque le complément de lieu est un argument. Par ailleurs, les GNev en position de lieu ne renvoient pas à des repères spatiaux spécifiés, en ce sens qu'ils ne renseignent pas directement sur le lieu de réalisation de l'événement. Ces particularités confirment la forme de spatialité non prototypique des GNev.

\subsection{Une localisation spatio-temporelle}

Contrairement aux GNobj, les GNev en complément de lieu comportent une spécification temporelle. La différence entre (31a) et (31b) est que dans (31b) le constituant requis syntaxiquement pour exprimer le lieu indique également une localisation dans le temps :

(31)a. Pierre va au Stade de France.

(31)b. Pierre va au match France-Argentine.

Cette spécification temporelle, non exigée syntaxiquement et relevant donc uniquement du GNev, explique la différence de conditions de vérité entre (32a) et (32b) :

(32)a. Sophie est au meeting.

(32)b. Sophie est sur le lieu du meeting.

Dans (32a), le moment où la localisation spatiale est observée coïncide avec la réalisation de l'événement dénoté. Tel n'est pas le cas dans (32b), où la localisation spatiale et l'actualisation de l'événement peuvent être disjoints temporellement. Les GNev en 
position de lieu ne désignent donc pas de purs repères spatiaux, comme le montre encore l'impossibilité de reprise anaphorique par cet endroit :

(33)a. Pierre va au Stade de France. Il aime beaucoup cet endroit.

(33)b. Pierre va au match France-Argentine. *Il aime beaucoup cet endroit.

Les repères spatiaux prototypiques - ceux qui sont à même de constituer des " endroits » - supposent l'abstraction de toute spécification temporelle. La prise en considération indépendamment de la variable temporelle est nécessaire à la constitution de purs localisateurs spatiaux.

L'imbrication des données spatiale et temporelle apparaît nettement lorsque les GNev ne sont pas contraints syntaxiquement à exprimer uniquement le lieu. En position d'adjoint, les GNev peuvent répondre à la fois aux questions Où ? et Quand ?:

(34) Les deux réalisateurs se sont rencontrés à la cérémonie des Oscars.

(35)a. Où les deux réalisateurs se sont-ils rencontrés ? - A la cérémonie des Oscars.

(35)b. Quand les deux réalisateurs se sont-ils rencontrés ? - A la cérémonie des

Oscars.

Les GNev en complément de lieu ne dénotent donc pas des sites spatiaux prototypiques. Leurs propriétés descriptives spatiales sont affectées par leurs propriétés descriptives temporelles.

\subsection{Sélection prépositionnelle}

Les contraintes qui pèsent sur la préposition introduisant le complément de lieu sont différentes selon que le site est dénoté par un GNobj ou un GNev. Les GNobj autorisent une large variété de prépositions, induisant des différences de configuration spatiale, qu'il s'agisse de localisation interne (à, dans, sur, etc.) ou externe (devant, près de, à gauche de, etc.) (cf. Borillo $1998: 82$ ) :

(36)a. La lampe est (dans / sur / en haut de / devant / à côté de / à gauche de)

l'armoire.

(36)b. Pierre est (à / dans / sous / à l'avant de / près de / derrière) la voiture.

Les GNev ne connaissent pas une telle diversité d'emplois. En complément de lieu, ils s'emploient principalement avec à, comme dans les exemples (6)-(7) et (31)-(34):

(37)a. Les journalistes sont (à / *à côté de / *derrière / *au bout de / *le long de / *autour de) un entraînement de l'équipe de France.

(37)b. Pierre est (à / *à proximité de / *à l'avant de / *en face de / *à gauche de / *au fond de) une vente aux enchères.

Les prépositions qui indiquent une configuration locative sont généralement exclues. L'absence d'orientation intrinsèque et de structure matérielle des sites événementiels bloque l'emploi de la plupart des locutions prépositionnelles formées à partir des «noms de localisation interne » (e.g. au fond de, à l'avant de, au bord de, en haut de, cf. Borillo 1988, Aurnague 1991, 1996, Huyghe 2005). La préposition à au contraire permet une localisation sans configuration, indiquant une simple coïncidence entre cible et site, sans véhiculer d'information sur la structure de l'entité repère ${ }^{3}$. Elle présente également l'avantage de convenir à la fois aux repérages spatial et temporel, avec le même schéma de localisation, et de laisser ainsi ouverte l'interprétation temporelle - qui peut être mobilisée lorsque le site est dénoté par un GNev. 
L'emploi des GNev avec d'autres prépositions spatiales n'est pas impossible. Il semble toutefois plus aléatoire, et dépend $\mathrm{du} \mathrm{GNev}$ et du prédicat en jeu. Ainsi certains GNev peuvent, sous certaines conditions, se construire avec dans:

(38)Où se sont-ils rencontrés ? - (Dans une fête / dans un bal de quartier / dans un colloque sur les adverbes / dans une compétition de kayak / dans un meeting des Verts / dans un mariage / dans une vente aux enchères / dans un festival de jazz).

La préposition dans, à la différence de à, met l'accent sur la description interne du site (cf. Katz 2002 : 41, Huyghe $2009: 205$ ). Cette description ne peut pas s'appliquer directement à l'étendue du site, les événements n'étant pas décrits comme des entités dotées d'extension spatiale (cf. (10b)). On peut cependant penser que le caractère collectif des événements dénotés dans (38), qui impliquent de multiples acteurs, contribue à la construction avec dans - les cibles étant alors présentées comme faisant partie de la collectivité qui définit l'événement. Reste que cette condition n'est pas suffisante, la construction dans + GNev étant soumise à des contraintes complexes. Outre que dans s'accommode nettement moins aisément que à des $\mathrm{GNev}$ définis (39), tous les $\mathrm{GN}$ dénotant des événements collectifs n'admettent pas la construction avec dans (40):

(39)a. Où se sont-ils rencontrés ? - (Au colloque sur les adverbes / au meeting des Verts / au bal de quartier).

(39)b. Où se sont-ils rencontrés ? - ??(Dans le colloque sur les adverbes / dans le meeting des Verts / dans le bal de quartier).

(40) Où se sont-ils rencontrés? - ??(Dans une avant-première / dans un enterrement / dans un entraînement de rugby / dans une inauguration de galerie d'art / dans une rétrospective Fritz Lang / dans un examen de rattrapage).

La compatibilité des GNev avec dans semble également dépendre du verbe qui introduit le constituant locatif. Ainsi les exemples (38) contrastent-ils avec (41) :

(41)a. ??Pierre et ses amis sont (dans un bal de quartier / dans une compétition de kayak).

(41)b. ??Sophie va (dans une vente aux enchères / dans un colloque sur les adverbes).

(41)c. ??Ils se rendent (dans un mariage / dans un meeting des Verts).

41 La distribution des GNev avec dans paraît donc beaucoup plus contrainte que celle avec $\grave{a}$. Elle semble à certains égards difficile à prédire - ou demanderait tout au moins une étude approfondie.

Il existe par ailleurs des GNev (très minoritaires) qui peuvent s'employer avec d'autres prépositions spatiales que à ou dans. Tel est le cas dans :

(42)a. Un barrage de police a été mis en place à proximité de l'accident.

(42)b. Une foule de gens se pressent à l'entrée de l'exposition

(42)c. Il y a plusieurs campings dans les environs du festival.

位 dans. Elles mettent en jeu une localisation externe et une configuration spatiale qui tendent à assimiler les événements sites à des objets du monde.

Notons, dans le même ordre d'idées, que certains GNev, lorsqu'ils se combinent avec des prépositions qui admettent les deux lectures spatiale et temporelle, semblent plutôt sélectionner l'interprétation spatiale. Ainsi la locution prépositionnelle de localisation interne au milieu de, qui a des emplois spatiaux et temporels (e.g. au milieu de la salle vs au milieu de la journée, cf. Gréa dans ce numéro), reçoit plutôt une lecture spatiale lorsqu'elle se construit avec des noms comme exposition, manifestation et embouteillage, d'où le 
contraste entre (43a), où le SP introduit par au milieu de a une interprétation temporelle, et $(43 b)$ :

(43)a. Pierre a fait un malaise au milieu de (la cérémonie / la visite / la conférence de presse).

(43)b. Pierre a fait un malaise au milieu de (l'exposition/ la manifestation /

l'embouteillage).

Certains noms d'événements ont donc une référence spatiale plus saillante que d'autres, et paraissent par là-même plus proches des noms d'objets.

Les GNev dans (42) et (43b) ne sont pas pour autant réductibles à des GNobj. Leurs propriétés événementielles ne sont pas neutralisées. La prédication événementielle reste accessible et compatible avec leur emploi comme complément de lieu :

(44) a. Un barrage de police a été mis en place à proximité de l'accident qui a eu lieu sur l'A25.

(44)b. Pierre a fait un malaise au milieu de la manifestation qui a eu lieu cet aprèsmidi, à Paris, contre le plan d'austérité gouvernemental.

Il n'y a donc pas, pour les noms comme accident et manifestation de polysémie stricto sensu qui consisterait en une double acception 'objet' et 'événement'. On peut considérer que ces noms sont lexicalement multitypés (cf. Pustejovsky 1995, Godard \& Jayez 1996) et qu'il leur correspond un type mixte 'objet-événement', ou bien qu'ils présentent deux facettes lexicales 'objet' et 'événement' (cf. Cruse 1996, Kleiber 1999a), voire tout simplement qu'ils décrivent des événements d'un type particulier, dotés de propriétés spatiales plus marquées que les autres. On reconnaîtra en tout cas les disparités existant entre les GNev, et le fait que certains GNev ont des propriétés descriptives qui les rapprochent des GNobj.

Deux points ressortent de l'ensemble de ces observations. D'une part, les GNev, en complément de lieu, ont des emplois beaucoup plus contraints que les GNobj, ce qui témoigne de la particularité de leurs propriétés descriptives spatiales. D’autre part, il existe entre les GNev des différences de dénotation spatiale, certains GNev décrivant des entités aux caractéristiques spatiales plus saillantes que d'autres. Nous reviendrons sur ce second point au $\$ 3$.

\subsection{Quand la localisation implique l'action}

La propriété la plus singulière des sites événementiels est l'absence de spécification spatiale. En effet, lorsqu'il est dénoté par un GNev, le lieu à proprement parler dépend des caractéristiques spatio-temporelles de l'événement mais n'est pas directement connu. Les phrases du type (6)-(7) ne spécifient pas le repère spatial visé, d'où la possibilité d'interroger sur la localisation, comme dans (45):

(45) - Pierre va à un colloque sur les adverbes.

- Où ça?

- A Paris.

50 Ce type d'interrogation est certes possible avec un site non événementiel, mais il s'agit alors de préciser la localisation dans l'espace, et non de l'identifier :

(46) - Pierre va à Paris.

- Où ça ? / Où précisément?

- Dans le XIIIe arrondissement.

Dans cet exemple, la relation entre les deux compléments de lieu successifs est une relation topologique d'inclusion - les deux compléments peuvent être liés par une relation partitive en de (le XIIIe arrondissement de Paris). Aucune relation de ce type n'est 
observée dans (45), en raison notamment de la non-congruence ontologique des sites, l'un étant un événement et l'autre un objet ${ }^{4}$.

En cas de désignation du site par un GNev, le lieu indiqué est donc le lieu de réalisation de l'événement. Ce lieu participe de l'individuation de l'événement dénoté, un événement particulier dans le monde étant notamment identifié par son ancrage spatio-temporel. Corollairement, les phrases du type (6)-(7) présupposent la spécificité de l'événement les GNev à interprétation générique en sont exclus.

Si les GNev en complément de lieu ne spécifient pas la localisation spatiale, on peut s'interroger sur leur rôle discursif et sur la pertinence informative des phrases qui les contiennent. Ces phrases servent en fait généralement à décrire l'action du sujet, en indiquant sa participation à un événement. Ainsi, en complément locatif de être, les GNev peuvent répondre à la question Que fait x ? :

(47) - Que fait Pierre ?

- Il est (à un concert / au match France-Argentine / à l'assemblée générale des copropriétaires).

Le complément de lieu événementiel permet d'indiquer l'action de la cible, à savoir sa participation à un événement collectif - le degré d'implication et le rôle précis de la cible dans l'événement étant sous-spécifiés. La nécessité d'une telle interprétation explique pourquoi les cibles non animées ne s'emploient avec être à + GNev :

(48 ) *Les sièges sont (à un concert / au match France-Argentine / à l'assemblée générale des copropriétaires).

Les GNev en position de lieu illustrent ainsi le lien sémantique, souvent relevé dans les travaux consacrés aux prépositions en et à, qui existe entre la localisation d'une entité et la détermination de son statut ou de son action (cf. Guillaume 1919, Vandeloise 1988, Franckel \& Lebaud 1991, Leeman 1995, Aurnague 2004, Haas 2009 inter alia). Vandeloise (1988) en particulier observe que la préposition à peut avec certains noms de lieux indiquer une « routine » fonctionnelle, à laquelle s'associe la cible :

(49) Pierre est (à l'école / à la fenêtre / à la plage / au gymnase / au piano).

Ces exemples indiquent une activité de la cible, en lien avec les propriétés fonctionnelles du site. Ils sont en cela proches de (6)-(7), dans lesquels la cible participe à une action dénotée par le complément de lieu. Les contraintes d'emploi et d'interprétation ne sont toutefois pas les mêmes dans les deux cas. D'une part, l'interprétation fonctionnelle avec un site non événementiel est contingente. Elle dépend du site sélectionné (e.g. (49) vs Pierre est (à Paris / à un endroit difficile à déterminer)), voire de la cible, comme le montre la comparaison de (49) et (50):

(50) Mon parapluie est (à l'école / au gymnase).

57 L'interprétation actionnelle avec les GNev, au contraire, s'impose, quel que soit le GNev employé - d'où la contrainte sur la cible illustrée dans (48). D'autre part, les phrases du type (49) nécessitent la présence de l'article défini ${ }^{5}$. D'une manière générale, la préposition $\grave{a}$, dans ses emplois purement spatiaux, est difficilement compatible avec l'article indéfini (cf. Lamiroy 1983, Vandeloise 1988). Le cas où le site est dénoté par un GNev constitue une exception notable à cette prédilection d'emploi, comme le remarque Vandeloise (1988 : 130). Ajoutons que pour les GNev sites, l'inférence actionnelle n'est pas réservée aux emplois avec à, mais se rencontre également avec dans, ce qui explique que l'on retrouve la contrainte sur le sujet humain:

(51)a. Pierre et ses collègues sont dans un colloque.

(51)b. ??Les sièges sont dans un colloque. 
La spécificité locative des GNev peut donc contribuer à expliquer leur prédilection d'emploi avec $\grave{a}$, en vertu des propriétés interprétatives de cette préposition, mais la préposition n'est pas à l'origine de l'interprétation actionnelle, contrairement à ce qui est le cas dans (49) - comparer (49) et Pierre est (dans l'école / sur la plage / dans le gymnase), qui, contrairement à (51a), n'implique aucune action de la cible.

\section{Hétérogénéité des GNev}

Les sites désignés par des GNev présentent de nombreuses particularités qui les distinguent des sites spatiaux prototypiques, tels qu'ils sont dénotés par les GNobj. Ces particularités confirment l'idée que les GNev ont des propriétés descriptives spatiales différentes de celles des GNobj. Les GNev présentent une autre différence significative avec les GNobj, qui est que tous ne peuvent pas s'employer comme complément de lieu. Aux GNev sous (52) s'opposent ceux sous (53) :

(52) réunion, concert, conférence de presse du ministre des affaires étrangères, mariage d'un collègue, avant-première du film, cérémonie en hommage aux victimes de la catastrophe, colloque sur les adverbes, festival de théâtre, manifestation contre le plan d'austérité, cocktail, fête, exposition Monet, vente aux enchères, entraînement de l'équipe de France, dîner de gala, office religieux, vernissage, grand prix automobile, congrès international de la critique, séance extraordinaire du conseil des ministres, enterrement, inauguration de la salle des fêtes, rétrospective Fritz Lang, session de rattrapage, meeting électoral, concours, visite de l'appartement, forum anti-mondialisation, communion de Pierre, réception, compétition de kayak, assemblée générale des copropriétaires, tirage au sort des gagnants, célébration œcuménique, procès d'assises, tournoi d'escrime

(53) éclipse, explication avec Vincent, explosion de la bombe, séisme, panne de voiture, suppression de cette aide publique, scène improbable, rupture du câble d'alimentation, drame, agonie du chef d'état, discussion animée, crash du Concorde, perfusion du patient, augmentation du prix du gaz, avalanche, déclenchement de la procédure d'urgence, crime,suicide de Vincent, agression, détournement de fonds, séparation du groupe, exploit du champion, découverte d'un vaccin, ajustement budgétaire, adhésion de Vincent au club alpin, arrangement, invasion de fourmis, diffamation, rupture idéologique, malentendu, démantèlement du consortium, suspension des expériences, miracle, aventure incroyable, décomposition du produit, déraillement du train,dispute,contamination des cellules souches ${ }^{6}$

60 En effet, seuls les GNev (52) peuvent s'employer dans les tournures suivantes :

(54)a. Pierre était à un / le + GNev.

(54)b. Pierre est allé à un / le + GNev.

(54)c. Où se sont-ils rencontrés ? - A un / le + GNev.

61 L'ensemble des GNev (53) récusent ces tests. Tel est le cas par exemple dans :

(55) *Pierre était (à l'éclipse / à une explication avec Vincent / à l'explosion de la bombe / au séisme / à une panne de voiture / à la suppression de cette aide publique / à une scène improbable / à la rupture du câble d'alimentation).

(56) *Pierre est allé (au drame / à l'agonie du chef d'état / à une discussion animée / au crash du Concorde / à une perfusion du patient / au déclenchement de la procédure d'urgence / à une avalanche / à l'augmentation du prix du gaz).

(57) Où se sont-ils rencontrés ? - *(A un crime / au suicide de Vincent / à un détournement de fonds / à la séparation du groupe / à une agression / à un exploit du champion / à la découverte d'un vaccin / à un ajustement budgétaire). 
On peut se demander ce qui distingue les GNev sous (52) de ceux sous (53). Nous tenterons de faire apparaître les propriétés permettant aux premiers de jouer le rôle de lieu, puis de mettre en évidence l'hétérogénéité référentielle des seconds.

\subsection{Evénements collectifs}

Les GNev (52) remplissent deux conditions : ils dénotent des événements (i) duratifs et (ii) programmés. La première condition implique que les GNev se construisent avec des expressions de durée, qu'il s'agisse de compléments de la forme de $x$ temps ou du prédicat durer $x$ temps,où $x$ temps est constitué d'un cardinal et d'un nom de mesure temporelle (cf. Gross \& Kiefer 1995, Godard \& Jayez 1996, Haas et al. 2008). Tel est le cas par exemple dans :

(58)a. (une réunion / un concert / une vente aux enchères / un meeting électoral) de trois heures

(58)b. (La conférence de presse du ministre des affaires étrangères / le dîner de gala / l'entraînement de l'équipe de France/ l'assemblée générale des copropriétaires) a duré trois heures.

La seconde condition implique que les événements soient organisés et prévus par l'ensemble des participants, et puissent faire l'objet d'une projection dans le temps. Ainsi les GNev (52) sont-ils compatibles avec des prédicats comme être prévu ou être reporté, accompagnés d'un complément de datation, comme dans :

(59)a. (Une rétrospective Fritz Lang / l'inauguration de la salle des fêtes / une session de rattrapage / la communion de Pierre) est prévu(e) le mois prochain.

(59)b. (Le concours / la cérémonie en hommage aux victimes de la catastrophe / le tirage au sort des gagnants / la fête) est reporté(e) au mois prochain.

Ces GNev dénotent ce que Gross et Kiefer appellent des événements «créés » (1995: 61-63), qui sont le fait de sujets humains agissant intentionnellement. Ils se caractérisent par leur compatibilité avec des verbes comme organiser, annuler, supprimer et des adjectifs comme improvisé, réussi, planifié, préparé (Gross \& Kiefer 1995: 62). En pratique, la dénotation d'événements programmés permet aux GNev (52) de se combiner avec des verbes de déplacement impliquant une destination (aller, se rendre, etc.), le fait de prévoir un événement étant nécessaire pour pouvoir s'y rendre.

La description d'une certaine stabilité temporelle, comprenant durée et projection dans le temps, est donc nécessaire pour qu'un GNev puisse jouer le rôle de lieu. Prises indépendamment, les deux conditions citées ne sont toutefois pas suffisantes, car il existe des GNev dans (53) qui peuvent satisfaire l'une ou l'autre - dispute par exemple renvoie à un événement duratif et démantèlement du consortium à un événement programmé. Une troisième condition peut être prise en compte, qui semble cruciale pour identifier les GNev sous (52). Programmés et duratifs, les événements décrits dans (52) sont tous collectifs, en ce sens qu'ils consistent en des rassemblements humains. Le fait que les participants soient réunis dans un but connu, à savoir la réalisation d'un événement planifié, permet d'installer cet événement dans l'espace et d'en faire un repère spatial pertinent. La pluralité des participants peut en outre expliquer l'association à un lieu étendu, et l'emploi avec dans commenté plus haut. 


\subsection{Evénements non contrôlés}

67 La catégorie des GNev qui ne peuvent pas jouer le rôle de lieu est elle-même hétérogène. On peut distinguer deux conditions suffisantes, non mutuellement exclusives, de l'appartenance à cette catégorie: la dénotation d'événements non contrôlés et la dénotation d'événements non physiques.

Les GNev sous (60) renvoient à des événements non contrôlés :

(60) séisme, explosion de la bombe, avalanche, crime, crash du Concorde, éclipse, rupture du câble d'alimentation, panne de voiture, drame, agression, disparition inquiétante, miracle, incident nucléaire, décès de Pierre, naissance, éclosion de l'œuf, réaction en chaîne, catastrophe, coup d'état, collision entre les deux véhicules,miracle, meurtre, révolte des prisonniers, violente secousse, effraction, choc frontal,délit mineur, aventure incroyable, invasion de fourmis, crevaison, propagation du virus

69 Ces GNev se distinguent des précédents par leur distribution avec se produire, qui exprime la réalisation d'un événement accidentel, non programmé par l'ensemble de ses participants (cf. Gaatone 1992, Gross \& Kiefer 1995, Gross 1996) :

(61) (L'avalanche / le crash du Concorde / l'explosion de la bombe / le crime / un miracle / une violente secousse / un incident nucléaire / l'effraction) s'est produit (e) en fin d'après-midi.

70 En effet, les GNev (52) ne sont pas compatibles avec se produire:

(62) *(La réunion / un concert / la conférence de presse du ministre / une cérémonie en hommage aux victimes de la catastrophe / une compétition de kayak / le vernissage / le concours / l'assemblée générale des copropriétaires) s'est produit(e) en fin d'après-midi.

71 Se produire se construit avec des sujets événementiels, tout en ayant un spectre d'emplois moins large que avoir lieu. Les GNev sélectionnés dénotent des événements «fortuits » (Gross \& Kiefer 1995 : 53-61), qui s'opposent aux événements programmés par le fait qu'ils échappent à la volonté (d'une partie au moins) de leurs participants. Il n'y a pas d'intentionnalité de la part de l'ensemble des acteurs de l'événement.

72 Non planifiés, les événements décrits dans (60) sont généralement constatés après coup, d'où l'emploi privilégié des GNev correspondants avec des verbes aux temps accomplis, comme cela a été relevé dans (25)-(28). En outre, beaucoup de GNev sous (60) décrivent des événements ponctuels (cf. Gross \& Kiefer 1995 : 55-56) et sont incompatibles avec les compléments et prédicats de durée :

(63)a. *un crime de deux heures, ${ }^{*}$ une naissance de six heures, ${ }^{*}$ un délit mineur de trois minutes, \#une disparition de deux jours ${ }^{7}$

(63)b. (*Le décès de Pierre / *la collision entre les deux véhicules / *le meurtre / \#la rupture du câble d'alimentation) a duré dix minutes.

73 On trouve notamment parmi ces noms d'événements les dérivés de verbes d'achèvement qui héritent de la structure aspectuelle de leur base, comme naissance, rupture, disparition, crevaison, etc. (cf. Haas et al. $2008:$ 2056). La description ponctuelle n'est pas nécessaire à la dénotation d'événements accidentels, certains événements fortuits étant duratifs (cf. Gross \& Kiefer 1995: 56). Elle semble en revanche suffire, tous les GNev ponctuels dénotant des événements non contrôlés.

L'absence de contrôle des participants sur les événements dénotés dans (60) fait que ceuxci ne constituent pas des repères spatiaux stables et efficaces. Il semble que moins un 
événement est contrôlé, stable et programmé dans le temps, moins il a de chances d'endosser le rôle de site spatial.

\subsection{Evénements non physiques}

Parmi les GNev qui ne peuvent pas s'employer comme complément de lieu, certains dénotent des événements non physiques, i.e. des événements qui n'impliquent pas directement des participants physiques. Tel est le cas des GNev dans :

(64) augmentation du prix du gaz, suppression de cette aide publique, ajustement budgétaire, détournement de fonds, prolongation du congé maternité, coïncidence, fusion entre les deux entreprises, cessation de paiement, adhésion de Vincent au club alpin, rupture idéologique, déclic, dévalorisation de l'université, mutation technologique, chute du système, renouvellement de la classe dirigeante, annulation de la procédure judiciaire, mélange des genres, renversement des valeurs, malentendu, suspension des expériences, rêve, hausse de la criminalité, démantèlement du consortium

76 Ces GNev ne désignent pas nécessairement des événements non contrôlés. Certains sont incompatibles avec se produire :

(65) ??(La suppression de cette aide publique / la prolongation du congé maternité / une fusion entre les deux entreprises / l'adhésion de Vincent au club alpin / une dévalorisation de l'université / un mélange des genres / une suspension des expériences) s'est produit(e) l'an dernier.

77 Les GNev sous (64) se distinguent de l'ensemble de ceux sous (52) et (60) par l'absence de référence spatiale saillante. Leur emploi comme cible spatiale paraitt moins favorisé. Il est certes permis de dire :

(66) Il y a eu (une augmentation du prix du gaz / une suppression de cette aide publique / un malentendu / une annulation de la procédure judiciaire / une suspension des expériences / un déclic / une rupture idéologique / un ajustement budgétaire) en France l'an dernier.

Cependant, il semble difficile de définir des lieux par la seule localisation de tels événements, les GNev (64) se construisant difficilement avec le nom lieu. Ainsi (68) contraste-t-il avec (9) et (67) :

(67) Pierre est retourné sur le lieu (du séisme / de l'avalanche / du crash du Concorde / du crime / de l'incident nucléaire / de la collision entre les deux véhicules / du drame / de l'explosion de la bombe).

(68) *Pierre est retourné sur le lieu (de l'augmentation du prix du gaz/ de la suppression de cette aide publique / du malentendu/ de l'annulation de la procédure judiciaire / de la suspension des expériences / du déclic / de la rupture idéologique / de l'ajustement budgétaire).

Les GNev (64) décrivent des événements dont la réalisation ne dépend pas crucialement d'un ancrage spatial. Il peut s'agir d'événements mentaux (rêve, déclic, malentendu), de changements idéologiques (renversement des valeurs, rupture idéologique), de procédures administratives (prolongation du congé maternité, adhésion de Vincent au club alpin) ou juridiques (annulation de la procédure judiciaire), d'opérations financières (cessation de paiement, détournement de fonds, démantèlement du consortium), etc. Ces événements impliquent des changements qui affectent des entités non physiques (idées, processus mentaux, personnes morales, sociétés, etc.), de sorte que leur localisation spatiale se présente comme non nécessaire ou secondaire. 
80 Les propriétés descriptives spatiales ne sont donc pas les mêmes pour tous les GNev. Corollairement, le critère spatial peut contribuer à la distinction entre différents types d'événements.

\section{Conclusion}

81 Tels qu'ils sont décrits par les GNev, les événements (i) sont des entités spatiales d'un type particulier, différent de celui associé aux GNobj, et (ii) n'ont pas tous la même relation à l'espace. S'agissant du premier point, nous avons montré que les GNev dénotent des entités qui font l'objet d'un mode de localisation spatiale spécifique et qui, en position de site, impliquent une spécification temporelle, tout en renseignant sur l'action de la cible. Les particularités descriptives spatiales des GNev tiennent en grande partie à leurs propriétés temporelles (condition sine qua non de leur dynamicité). Les entités spatiales prototypiques sont celles dénotées par les GNobj, qui n'impliquent pas de description temporelle et sont donc à même de décrire un espace "pur ", i.e. conçu en dehors de toute variable temporelle.

82 Le second point implique que la dénotation spatiale varie selon les GNev. Nous pouvons à ce stade distinguer trois sous-classes, selon que les GNev dénotent :

- des événements collectifs, programmés et duratifs, qui sont localisés dans l'espace et peuvent jouer le rôle de lieu (e.g. une réunion),

- des événements non contrôlés, localisés dans l'espace mais ne pouvant pas jouer le rôle de lieu (e.g. un crime),

- des événements non physiques, qui ne dépendent pas directement ni nécessairement de l'espace (e.g. un malentendu).

Ces sous-classes ne sont pas elles-mêmes homogènes. Par exemple, certains noms relevant de la première catégorie ont des propriétés spatiales plus saillantes que d'autres (e.g. manifestation vs réunion, cf. §2.2.), ce qui suggère l'existence de distinctions plus fines que celles esquissées ici, voire d'un continuum de la spatialité dans le domaine événementiel.

$84 \mathrm{Si}$, comme on le considère souvent, l'espace est un critère de distinction entre entités concrètes et abstraites, et la description d'entités spatiales un critère de distinction entre noms concrets et noms abstraits, alors les noms d'événements intègrent différents degrés d'abstraction et se trouvent, par leur hétérogénéité, à la charnière entre noms concrets et noms abstraits. Certains noms d'événements sont, en ce sens, plus "concrets» que d'autres, et plus proches que d'autres des noms d'objets.

Un autre enseignement de cette étude concerne les relations entre espace et temps dans la constitution des événements, tels qu'ils sont décrits par les GNev. Si, d'une part, les propriétés spatiales des événements sont déterminées par leurs propriétés temporelles et si, d'autre part, tous les événements n'ont pas la même relation à l'espace, alors qu'ils ont tous une relation fondamentale au temps, alors la composante temporelle dans la structure spatio-temporelle des événements est l'élément dominant. Il n'est pas juste de considérer que les événements n'ont pas de relation directe à l'espace, mais il est également erroné de penser que les propriétés spatiales et les propriétés temporelles des événements sont d'égale importance. Le temps est pour les événements l'élément structurel principal, et celui qui en dernier ressort les distingue des objets. 
86

ans des travaux ultérieurs, nous tâcherons d'élargir le corpus des GNev étudiés, et d'affiner les critères de distinction entre les différents GNev. Nous nous interrogerons également sur les liens entre l'autonomie syntaxique des noms d'événements et leurs propriétés de description spatiale, en nous demandant si le degré de dépendance d'un nom événementiel avec ses éventuels arguments est corrélé à son aptitude à dénoter des entités spatiales.

\section{BIBLIOGRAPHIE}

Aurnague, M., 1991. Contribution à l'étude de la sémantique formelle de l'espace et du raisonnement spatial : la localisation interne en français, sémantique et structures inférentielles. Thèse de doctorat. Toulouse : Université Paul Sabatier.

Aurnague, M., 1996. «Les noms de localisation interne : tentative de caractérisation sémantique à partir de données du basque et du français », Cahiers de lexicologie 69, 159-192.

Aurnague, M., 2004. Les structures de l'espace linguistique. Regards croisés sur quelques constructions spatiales du basque et du français. Louvain / Paris : Editions Peeters.

Balibar-Mrabti, A., 1990. « Analyse d'adverbes en dans », Langue Française 86, 65-74.

Berthonneau, A.-M., 1989. Composantes linguistiques de la référence temporelle. Les compléments de temps, du lexique à l'énoncé. Thèse de doctorat d'état. Paris : Université de Paris VII.

Borillo, A., 1988. « Le lexique de l'espace : les noms et les adjectifs de localisation interne », Cahiers de grammaire 13, 3-22.

Borillo, A., 1998. L'espace et son expression en français. Paris : Ophrys.

Casati, R. \& Varzi, A., 1999. Parts and Places. The Structures of Spatial Representation. Cambridge, Mass. : The MIT Press.

Corblin, F., (dans ce numéro). « Locus et telos : aller à l'école, être à la plage », Corela.

Cruse, A., 1996. « La signification des noms propres de pays en anglais », in S. Rémi-Giraud \& P. Rétat (eds), Les mots de la nation. Lyon : Presses universitaires de Lyon, 93-102.

Davidson, D., 1967 [1993]. « La forme logique des phrases d'action », in D. Davidson, Actions et événements. Paris : PUF, 149-198.

Flaux, N. \& Stosic, D., 2011. « Noms d'idéalités, prépositions et temporalité », inE. Arjoca-Ieremia, C. Avezard-Roger, J. Goes, E. Moline \& A. Tihu (eds), Temps, aspect et classes de mots : études théoriques et didactiques. Arras : Artois Presses Université, 155-177.

Franckel, J.-J. \& Lebaud, D., 1991. « Diversité des valeurs et invariance du fonctionnement de en préposition et préverbe », Langue Française 91, 56-79.

Franckel, J.-J., 1993. « Il y a lieu de prendre place dans un endroit facilement localisable », in L. Danon-Boileau \& J.-L. Duchet (eds), Opérations énonciatives et interprétation de l'énoncé. Mélanges offerts à Janine Bouscaren. Paris : Ophrys, 209-221. 
Gaatone, D., 1992. « Les verbes événementiels et les dictionnaires : quelques observations ", in A. Clas (ed), Le mot, les mots, les bons mots. Hommage à Igor A. Mel'cuk à l'occasion de son soixantième anniversaire. Montréal : Presses de l'Université de Montréal, 95-108.

Godard, D. \& Jayez, J., 1996. « Types nominaux et anaphores : le cas des objets et des événements ", in W. De Mulder, L. Tasmowski-De Ryck \& C. Vetters (eds), Anaphores temporelles et (in-)cohérence, Cahiers Chronos 1. Amsterdam : Rodopi, 41-58.

Gréa, P., (dans ce numéro). « Le centre n'est pas au milieu (et inversement). Pour une approche phénoménologique et gestaltiste de la localisation », Corela.

Gross, G., 1996. « Prédicats nominaux et compatibilité aspectuelle », Langages 121, 54-72.

Guillaume, G., 1919 [1975]. Le problème de l'article et sa solution dans la langue française. Québec / Paris : Presses de l'Université Laval / Nizet.

Haas, P., 2009. Comment l'aspect vient aux noms. Les propriétés aspectuelles des noms à l'épreuve des restrictions de sélection imposées par certaines prépositions. Thèse de doctorat. Villeneuve-d'Ascq : Université de Lille 3.

Haas, P., Huyghe, R. \& Marín, R., 2008. « Du verbe au nom : calques et décalages aspectuels ", in J. Durand, B. Habert \& B. Laks (eds), Congrès Mondial de Linguistique Française - CMLF'08. Paris : Institut de Linguistique Française, 2051-2065.

Hacker, P.M.S., 1982. « Events and Objects in Space and Time », Mind 91, 1-19.

Huyghe, R., 2005. « Zones et parties : l'hétérogénéité des noms de localisation interne », Le Français Moderne 2/2, 184-211.

Huyghe, R., 2009. Les noms généraux d'espace en français. Enquête linguistique sur la notion de lieu . Bruxelles : De Boeck Duculot.

Huyghe, R., 2011. « La dénotation spatiale des noms d'événements », Lingvisticae Investigationes 34/1, 138-155.

Katz, E., 2002. «Systématique de la triade spatiale à, en, dans », Travaux de Linguistique 44, 35-49.

Kleiber, G., 1981. « Relatives spécifiantes et relatives non spécifiantes », Le Français Moderne 49/3, 216-233.

Kleiber, G., 1999a. Problèmes de sémantique. La polysémie en questions. Villeneuve d'Ascq : Presses Universitaires du Septentrion.

Kleiber, G., 1999b. « Anaphore associative et relations partie-tout : condition d'aliénation et principe de congruence ontologique », Langue Française 122, 70-100.

Kleiber, G., Benninger, C., Biermann Fischer, M., Gerhard Krait, F., Lammert, M., Theissen, A. \& Vassiliadou, H., (à paraître). «Typologie des noms : le critère se trouver + SP loc », Scolia.

Lamiroy, B., 1983. Les verbes de mouvement en français et en espagnol. Etudes comparées de leurs infinitives. Amsterdam / Philadelphie : John Benjamins Publishing Company.

Leeman, D., 1995. « Pourquoi peut-on dire Max est en colère mais non *Max est en peur ? ", Langue Française 105, 55-69.

Pustejovsky, J., 1995. The Generative Lexicon. Cambridge, Mass. : The MIT Press.

Quinton, A., 1979. « Objects and events », Mind 88, 197-214.

Spang-Hanssen, E., 1963. Les prépositions incolores du français moderne. Copenhague : GEC Gads Forlag. 
Vandeloise, C., 1996. « La méronomie, l'inclusion topologique et la préposition dans », Faits de Langue 7, 81-90.

Vandeloise, C., 1988. « Les usages spatiaux de la préposition à », Cahiers de Lexicologie 53, 119-148.

Vandeloise, C., 2001. Aristote et le lexique de l'espace. Rencontres entre la physique grecque et la linguistique cognitive. Stanford : CSLI Publications.

Van de Velde, D., 2006. Grammaire des événements. Villeneuve-d'Ascq : Presses Universitaires du Septentrion.

Vendler, Z., 1967. «Facts and Events », in Z. Vendler, Linguistics in Philosophy. Ithaca, N.Y. : Cornell University Press, 122-146.

\section{NOTES}

1. Seuls seront considérés ici comme GNev ceux qui se construisent avec avoir lieu. Ce critère exclut les « événements faibles » (Godard \& Jayez 1996), dénotés par des noms comme symphonie, comédie, chanson. Ces noms sont considérés comme noms d'événements en vertu de leur compatibilité avec les verbes aspectuels (cf. Gross \& Kiefer 1995), les constructions nominales de durée et les prépositions temporelles (cf. Berthonneau 1989). Ils ne sont cependant pas compatibles avec avoir lieu. Leur caractère véritablement événementiel est discuté dans Flaux \& Stosic (2011).

2. Dans la terminologie de Kleiber (1981), les prédicats « spécifiants » s'opposent aux prédicats «non spécifiants » par l'implication d'un ancrage spatio-temporel (e.g. entrer vs être blond). Seuls les prédicats spécifiants, parce qu'ils peuvent garantir l'individuation du sujet, autorisent les sujets indéfinis à interprétation spécifique et existentielle (i.e. non partitive) (cf. Un homme est entré vs *Un homme est blond).

3. Selon Spang-Hanssen, la préposition à « marque le lieu de façon ponctuelle » (1963: 183). Elle met donc en relation cible et site sans indication de configuration spatiale. De fait, à neutralise la description interne du site, qui est plutôt appréhendé de l'extérieur, contrastant potentiellement avec d'autres espaces de localisation (cf. Katz 2002 : 41, Huyghe 2009 : 205-207). Comme le montre Vandeloise (1988), il existe d'autres conditions, non spatiales, de l'emploi de à (cf. §2.3.). L'absence de configuration reste néanmoins un élément clé de la distinction entre à et les autres prépositions spatiales, qui spécifient la forme de la relation spatiale établie entre la cible et le site.

4. L'inclusion topologique, telle qu' « un ensemble A est inclus dans un ensemble B si tous les éléments de A sont des éléments de B » (Vandeloise 1996 : 84), met en jeu une relation de partietout, impliquant que les deux ensembles considérés relèvent du même type ontologique (cf. Kleiber 1999b). Tel est le cas dans (46), mais pas dans (45). Par ailleurs, la demande de précision exige que l'on focalise sur une partie du tout préalablement mentionné, ce qui ne peut en aucune manière être le cas dans (45), Paris ne désignant pas une partie du colloque.

5. L'interprétation même de cet article fait débat. De nombreux auteurs, à la suite de Vandeloise (1988 : 141), le considèrent comme un défini générique. Pour d'autres, comme Corblin (dans ce numéro), il s'apparente à un défini associatif. On s'accorde néanmoins à dire qu'il ne s'agit pas d'un défini spécifique standard, ce qui constitue une autre différence avec les emplois de à + GNev. En effet, l'interprétation de l'article défini dans des phrases comme Pierre est (au concert de Kurt Wagner / à l'assemblée générale des copropriétaires / au mariage de son collègue) est nécessairement spécifique, comme nous l'avons vu plus haut.

6. Les GN sont listés ici sans déterminant, celui-ci pouvant varier selon les tests spatiaux appliqués dans (54) et suivant la forme de chaque GN. 
7. Une interprétation durative est possible lorsque la durée porte sur un état transitoire résultant de l'événement (et non sur l'événement lui-même). Une disparition de deux jours et une rupture du câble d'alimentation de dix minutes sont tout à fait acceptables si on évalue le temps qu'a duré la phase stative suivant l'événement, pendant laquelle telle personne est disparue ou le câble est rompu. Les deux expressions sont cependant difficiles à former si la durée porte sur le processus conduisant aux états en question : la phase dynamique reste conçue comme ponctuelle.

\section{RÉSUMÉS}

Cet article traite des propriétés de description spatiale des GN dénotant des événements [GNev]. Les GNev se distinguent des GN dénotant des êtres ou des objets par le fait qu'ils ne désignent pas des cibles et des sites spatiaux prototypiques, en raison de leurs propriétés aspectuo-temporelles. En complément de lieu notamment, ils impliquent une localisation temporelle et la participation dynamique de la cible à l'événement dénoté. Le critère spatial fait également apparaître l'hétérogénéité référentielle des GNev, étayant la distinction entre des événements programmés et collectifs (une réunion), des événements non contrôlés (un crime) et des événements non physiques (un malentendu).

This paper deals with the spatial properties of NPs denoting events in French [ENPs]. Unlike NPs denoting beings or objects, ENPs do not refer to prototypical spatial entities, because of their aspecto-temporal properties. When used as place complements, they involve a temporal location and the dynamic participation of the located entity to the event. The spatial criterion also reveals the heterogeneity of ENPs, suggesting a distinction between planned and collective events (une réunion 'a meeting'), uncontrolled events ( un crime 'a crime') and non-physical events ( un malentendu 'a misunderstanding').

\section{INDEX}

Mots-clés : événement, espace, temps, localisation, complément de lieu

Keywords : event, space, time, location, place complement

\section{AUTEUR}

RICHARD HUYGHE

Université Paris Diderot - Paris 7 